\title{
Relationship between Nocturnal Blood Pressure and Left Ventricular Hypertrophy in Hypertensive Patients
}

Hang Zhu, Wei Zhang, Yajun Shi, Yiming Ma, Yunfeng Han, Jie Liu and Hao Xue

Department of Cardiology, Chinese People's Liberation Army General Hospital, 28 Fuxing Road, Beijing 100853, People's Republic of China

*Corresponding author: Hao Xue, Department of Cardiology, Chinese People's Liberation Army General Hospital, 28 Fuxing Road, Beijing, People's Republic of China, Tel: 8610 55499211; Fax: 8610 55499411; E-mail: xuehao301@hotmail.com

Rec date: April 16, 2014, Acc date: May 26, 2014, Pub date: June 07, 2014

Copyright: ( $) 2014$ Zhu H, et al. This is an open-access article distributed under the terms of the Creative Commons Attribution License, which permits unrestricted use, distribution, and reproduction in any medium, provided the original author and source are credited.

\begin{abstract}
Objective: Left ventricular hypertrophy $(\mathrm{LVH})$ is the most common target organ damage in hypertension. However, the association of LVH and circadian rhythm of blood pressure (BP) is unknown. The objective of the present study was to explore the relationship between circadian rhythm of BP and LVH using ambulatory blood pressure monitoring (ABPM).
\end{abstract}

Study design: A total of 325 untreated hypertensive patients were recruited. The patients were divided into two groups: hypertensive patients with LVH $(n=121)$ and without LVH $(n=204)$. Twenty-four-hour ABPM was performed in all the patients to collect the following parameters: 24-hour average systolic and diastolic pressure, daytime average systolic and diastolic pressure, nocturnal average systolic and diastolic pressure, and night to day BP ratio. The relationship between LVH and the various ABPM parameters was analyzed.

Results: We found that the average nocturnal systolic blood pressure (SBP) in hypertensive patients with LVH was higher than that in hypertensive patients without LVH $(145 \pm 16.1 \mathrm{mmHg}$ versus $136 \pm 12.7 \mathrm{mmHg}, \mathrm{P}<0.05)$. The average night to day SBP ratio in hypertensive patients with LVH was also higher than that in hypertensive patients without $\mathrm{LVH}(0.93 \pm 0.04$ versus $0.86 \pm 0.04, \mathrm{P}<0.05)$. Multiple regression analysis indicate that the average nocturnal SBP and the night to day SBP ratio were associated with LVH (Odds ratio (OR) 1.67, 95\% Cl: 1.31-3.21; OR: $1.88,95 \% \mathrm{Cl}: 1.56-3.78$ ) by adjustment for traditional covariates.

Conclusion: The average nocturnal SBP and the average night-day SBP ratio are independent risk factors of LVH in patients with hypertension.

Keywords: Hypertension; Left ventricular hypertrophy; Nocturnal blood pressure; Ambulatory blood pressure monitoring

\section{Introduction}

The most common target organ damage associated with hypertension is left ventricular hypertrophy (LVH) [1-3]. An epidemiologic study revealed that the incidence rate of $\mathrm{LVH}$ in hypertension in the Chinese population is up to $42.7 \%$ [4]. Previous research has shown that $\mathrm{LVH}$ is an independent risk factor for cardiovascular and cerebrovascular diseases (CCVD) including coronary heart disease, heart failure, and stroke [5-8]. Early detection, diagnosis and treatment of LVH are significantly important for the prevention of CCVD in patients with hypertension.

Although hypertension is a major cause of $\mathrm{LVH}$, the degree of $\mathrm{LVH}$ is not in parallel with clinical blood pressure (CBP) [9]. CBP is influenced by temporary physical and mental conditions, which has contingency and short-term variability. So it cannot necessarily reflect the BP fluctuation amplitude, average BP level over a period of time and circadian rhythm of BP. However, current clinical research, evidence-based results and recommended guidelines for hypertension diagnosis and treatment recommendations are based on average value CBP, and rarely take into account the correlation between circadian rhythm of blood pressure and target organ damage. Ambulatory blood pressure monitoring (ABPM) can measure blood pressure within 24 hours, including 24-hour average BP, daytime and nocturnal average BP and night to day BP ratio. Now, the association between circadian rhythm of blood pressure status with left ventricular hypertrophy (LVH) and its therapeutic implications are still inconsistent [10-12]. To address this issue, we explore the relationship between circadian rhythm of blood pressure and LVH in patients with hypertension. The results may provide the timing of drug administration for intervention of target organ damage in hypertension and prevention of hypertension-related cardiovascular diseases and death.

\section{Subjects and Methods}

\section{Subjects}

A total 989 consecutive untreated hypertensive patients (45 to 65 years of age) were continuously recruited from outpatients of the Chinese People's Liberation Army General Hospital between September 2009 and June 2012 in this study. ABPM (24-hour) was performed in 325 patients. The inclusion criteria were based on the 1999 World Health Organization International Society of Hypertension (WHO-ISH) Guidelines and risk classification standard [13], in which hypertension was defined as systolic blood pressure (SBP) $\geq 140 \mathrm{mmHg}$ and/ or diastolic blood pressure $\geq 90 \mathrm{mmHg}$. The exclusion criteria included secondary arterial hypertension, stroke 
Page 2 of 5

atria-ventricular conduction block, chronic obstructive bronchitis, valvular heart disease, hypertrophy cardiomyopathy, heart failure, diabetes, obesity (BMI $>28 \mathrm{~kg} / \mathrm{m} 2)$, pulmonary hypertension, coronary heart disease or other severe medical illnesses. All participants were Han nationality. The study was approved by the Ethics Committee of the Chinese People's Liberation Army General Hospital. The study was performed according to the guidelines of Helsinki Declaration. Informed consent was obtained from all patients.

\section{Clinical data collection}

A complete clinical date was obtained from all patients, including age, gender, heart rate (HR), weight, systolic blood pressure (SBP), diastolic blood pressure (DBP), family history of hypertension, diabetes mellitus, chronic kidney dysfunction(CKD), hyperlipidemia, previous myocardial infarction (MI), previous stroke. The following conventional cardiovascular risk factors were also recorded, including alcohol intake, cigarette smoking, and body mass index (BMI). BMI was calculated by using the formula of weight $(\mathrm{kg}) /$ height $\left(\mathrm{m}^{2}\right)$. Diabetes was diagnosed as fasting blood glucose $\geq 7.8 \mathrm{mmol} / \mathrm{L}$ or twohour postprandial blood glucose $\geq 11.1 \mathrm{mmol} / \mathrm{L}$.

\section{Biochemical variables determination}

Blood sample collection after overnight fasting. Biochemical parameters including serum total cholesterol (TC), total triglyceride (TG), high density lipoprotein cholesterol (HDL-C), low density lipoprotein cholesterol (LDL-C), creatine kinase (CK), creatine kinase isozyme (CK-MB), troponin T (TNT), blood glucose (GLU), urea nitrogen (BUN), and creatine (Cr) were determined using an automatic biochemical analyzer (Roche modular 7600 automatic biochemistry analyzer). The Chinese Modification of Diet in Renal Disease (C-MDRD) equation was used to estimate the estimated glomerular filtration rate eGFR:

eGFR $\left.\left[\mathrm{ml} / \mathrm{min} \cdot 1.73 \quad \mathrm{~m}^{2}\right)\right]=175 \quad \times \quad$ standardized $\quad \mathrm{Scr}$ $-1.154 \times$ age $0.203 \times 0.742$ (if female) [14]. eGFR $<60 \mathrm{ml} /\left(\mathrm{min} \cdot 1.73 \mathrm{~m}^{2}\right)$ was defined as renal dysfunction.

\section{Ambulatory BP Measurement}

24-hour ABPM: The study used American Sun Tech OSCAR 2 24hour ambulatory blood pressure monitor, with a cuff of $22 \mathrm{~cm} \times 12 \mathrm{~cm}$ length around the subject's left upper arm. The cuff was aerated at $40 \sim 220 \mathrm{mmHg}$ and the outgassing rate was $2 \mathrm{mmHg} / \mathrm{s}$. ABPM time was 24 hours and the machine automatically measured blood pressure once at a 30-minute interval in the day (6:00 22:00), and once at a 60minute interval at night (22:00 6:00). Monitoring started from 8 a.m. and ended at $8 \mathrm{a} . \mathrm{m}$. in the next day. Monitoring failure was defined as $\mathrm{SBP}<70 \mathrm{mmHg}$ or $\mathrm{SBP}>260 \mathrm{mmHg}, \mathrm{DBP}<40 \mathrm{mmHg}$ or $>150 \mathrm{mmHg}$.

Records of 24-hour ABPM parameters: The following ABPM parameters were determined: 24-hour average systolic and diastolic pressure (24 h SBP, 24 h DBP); average daytime (6:00-22:00) systolic and diastolic pressure (daytime SBP, daytime DBP); nocturnal (22:00-6:00) average systolic and diastolic pressure (nocturnal SBP, nocturnal DBP). Night to day blood pressure ratio was defined as mean nocturnal $\mathrm{BP} /$ mean daytime $\mathrm{BP}$ from 24 -hour ambulatory blood pressure recordings. A SBP dip was greater or equal to $10 \%$. Dipping pattern was classified as normal dipper $(0.8<$ night-day blood pressure ratio $\leq 0.9$ ) and non-dippers (night-day blood pressure ratio $>0.9$ ) [15].

\section{Echocardiography measurement}

Echocardiography operation method: Color Doppler Ultrasonic Diagnostic Apparatus (SEQUOIA512, American Siemens Co., Ltd) at the frequency of $3.5 \sim 5 \mathrm{MHz}$ was used for echocardiography. The following parameters were measured on the level of chordate tendineae of mitral valve by the parasternal long-axis plane when the patients were in the left-lateral position: Left ventricular end-diastolic dimension (LVEDD), left ventricular end-systolic dimension, (LVESD), interventicular septal thickness (IVST), posterior wall thickness (PWT), from which left ventricular ejection fraction (EF) and fractional shortening (FS) were calculated. Cardiac cycles were continuously detected to measure their mean values.

Parameters calculated with the formulas [16]: Left ventricular weight or left ventricular mass $(\mathrm{LVM})=0.8 \times 1.04$ [(IVSd+LVIDD +PWTd) 3-LVIDD3] + 0.6. Calculation of left ventricular mass index (LVMI): LVM/height ${ }^{2.7}$. LVH was defined as for male LVMI $>49.2$ $\mathrm{g} / \mathrm{m}^{2.7}$ and for female LVMI $>46.7 \mathrm{~g} / \mathrm{m}^{2.7}$.

\section{Statistical analysis}

All data were entered into Epidata database by specifically-assigned persons. All statistical analyses were performed with the SPSS13.0 statistical package (SPSS Inc, Chicago). Quantitative data was expressed by mean \pm standard deviation (SD). All categorical variables were presented as numbers and percentages. A chi-squared (X2) test was used in testing categorical variables. Differences in baseline levels of characteristics between the two groups were tested using the Student's t-test (t-test).

Multiple regression analysis was used to analyze the association ABPM parameters with $\mathrm{LVH}$ adjusting for cardiovascular risk factors including age, gender, BMI, BP, GLU, eGFR, TC, HDL-C, LDL-C and TG. $\mathrm{P}<0.05$ was considered as statistically significant.

\section{Results}

\section{Baseline clinical characteristics}

Baseline clinical characteristics between hypertensive patients with $\mathrm{LVH}$ and without LVH compared are shown in Table 1. Among the 325 patients with hypertension, 121 patients were concomitant with LVH and 204 patients were not concomitant with LVH based on LVMI. No significant differences was found in age, gender, systolic and diastolic pressure, blood glucose, cholesterol, triglyceride, low density lipoprotein cholesterol (LDL-C), estimation of glomerular filtration rate (eGFR) and high density lipoprotein cholesterol (HDLC) between hypertensive patients with and without $\mathrm{LVH}(\mathrm{P}>0.05)$. The BMI in hypertensive patients with LVH was significantly higher than that in hypertensive patients without LVH $(\mathrm{P}<0.05)$ (Table 1).

\begin{tabular}{|l|l|l|}
\hline \multicolumn{1}{|c|}{ Characteristics } & $\begin{array}{l}\text { Hypertension } \\
\text { without LVH }\end{array}$ & Hypertension with LVH \\
\cline { 2 - 3 } & $\mathrm{n}=204$ & $\mathrm{n}=121$ \\
\hline Age $\mathrm{Y}$ & $49.4 \pm 9.2$ & $51.6 \pm 10.2$ \\
\hline Men $\%$ & $47.8 \%$ & $48.4 \%$ \\
\hline BMl $(\mathrm{kg} / \mathrm{m} 2)$ & $24.3 \pm 3.1$ & $26.8 \pm 3.09^{*}$ \\
\hline SBP $\mathrm{mmHg}$ & $153.0 \pm 11.3$ & $156.1 \pm 11.9$ \\
\hline
\end{tabular}


Citation: Zhu H, Zhang W, Shi Y, Ma Y, Han Y, Liu J, Hao Xue (2014) Relationship between Nocturnal Blood Pressure and Left Ventricular Hypertrophy in Hypertensive Patients. J Hypertens 3: 151. doi:10.4172/2167-1095.1000151

Page 3 of 5

\begin{tabular}{|l|l|l|}
\hline DBP $\mathrm{mmHg}$ & $98.6 \pm 8.1$ & $99.8 \pm 8.4$ \\
\hline HDL-C mmol/L & $1.18 \pm 0.34$ & $1.08 \pm 0.51$ \\
\hline LDL-C mmol/L & $3.12 \pm 0.67$ & $3.21 \pm 0.71$ \\
\hline TC mmol/L & $5.09 \pm 0.98$ & $5.17 \pm 1.21$ \\
\hline TG mmol/L & $1.36 \pm 0.81$ & $1.52 \pm 0.88$ \\
\hline Glucose mmol/L & $5.06 \pm 0.92^{*}$ & $5.13 \pm 1.02$ \\
\hline eGFR ml/min per $1.73 \mathrm{~m}^{2}$ & 83 & 79 \\
\hline Smoking \% & 24 & 25.1 \\
\hline Drinking \% & 19.2 & 20.7 \\
\hline
\end{tabular}

Table1: Clinical Characteristics

Abbreviations: BMI: Body Mass Index; BP: Blood Pressure; SBP: Systolic Blood Pressure; DBP: Diastolic Blood Pressure; HDL-C: High Density Lipoprotein Cholesterol; LDL-C: Low Density Lipoprotein Cholesterol; TC: Total Cholesterol; TG: Triglyceride ; eGFR: estimated Glomerular Filtration Rate

Smoking was classified as average one pack of cigarettes per week over one year.

Drinking was classified as average $>500 \mathrm{ml}$ white or red wine per week over two years.

Clinical characteristics of age, BMI, SBP, DBP, glucose, HDL-C, LDL-C, TG and TC values are given as mean (SD); categorical variables are presented as percentages.

$\mathrm{P}<0.05$ with statistical significance. ${ }^{\star} \mathrm{P}<0.05$, vs control

\section{AMBP variables in hypertensive patients with and without LVH}

The nocturnal average SBP $(145 \pm 16.1 \mathrm{mmHg})$ in hypertensive patients with $\mathrm{LVH}$ was higher than that in hypertensive patients without LVH $(136 \pm 12.7 \mathrm{mmHg}, \mathrm{P}<0.05)$. The average night-day systolic pressure ratio $(0.93 \pm 0.04)$ in hypertensive patients with $\mathrm{LVH}$ was also higher than that in hypertensive patients without LVH $(0.86 \pm$ $0.04, \mathrm{P}<0.05$ ). The percentage of non-dipper (night-day blood pressure ratio $>0.9)$ in the LVH group was higher than that in the non-LVH group (68\% versus $46 \%$ ). No statistical significance was found in the following parameters: 24-hour SBP, 24-hour DBP, daytime SBP, daytime DBP, nocturnal SBP, nocturnal DBP and daytime-nocturnal SBP. The previous results suggested that the nocturnal SBP could increase the patients with hypertension concomitant with LVH (Table 2).

\begin{tabular}{|l|l|l|}
\hline \multicolumn{1}{|c|}{ Variables } & $\begin{array}{l}\text { Hypertension } \\
\text { without LVH }\end{array}$ & $\begin{array}{l}\text { Hypertension with } \\
\text { LVH }\end{array}$ \\
\cline { 2 - 3 } & $\mathrm{n}=204$ & $\mathrm{n}=121$ \\
\hline 24-h BP $(\mathrm{mmHg})$ & $146 \pm 20.1$ & $149 \pm 22.3$ \\
\hline SBP $(\mathrm{mmHg})$ & $94 \pm 21.4$ & $96 \pm 20.8$ \\
\hline DBP $(\mathrm{mmHg})$ & \multicolumn{2}{|l}{} \\
\hline Daytime blood pressure & \\
\hline
\end{tabular}

\begin{tabular}{|l|l|l|}
\hline SBP $(\mathrm{mmHg})$ & $155.7 \pm 12.6$ & $156 \pm 11.9$ \\
\hline DBP $(\mathrm{mmHg})$ & $99.2 \pm 10.3$ & $102.7 \pm 9.4$ \\
\hline \multicolumn{2}{|l|}{ Nighttime blood pressure } & $145 \pm 16.1^{* *}$ \\
\hline SBP $(\mathrm{mmHg})$ & $136 \pm 12.7$ & $90 \pm 14.4$ \\
\hline DBP $(\mathrm{mmHg})$ & $88 \pm 10.5$ & $0.93 \pm 0.04^{* *}$ \\
\hline Night-to-day blood pressure ratio & $0.87 \pm 0.03$ \\
\hline SBP & $0.86 \pm 0.04$ & $0.88 \pm 0.03$ \\
\hline DBP & 0.03
\end{tabular}

Table 2: 24 hour Ambulatory Systolic Blood Pressure variables in hypertensive patients with and without LVH

\section{Association between AMBP variables and LVH}

Multiple regression analysis was used to analyze the association ABPM parameters with LVH adjusting for age, gender, BMI, GLU, eGFR, TC, HDL-C, LDL-C and TG. We found that the average nocturnal systolic pressure and the night to day systolic pressure ratio were the risk factors of $\mathrm{LVH}$ (odds ratio (OR): 1.67, 95\% CI: 1.31-3.21; OR: $1.88,95 \%$ CI: 1.56-3.78) (Table 3).

\begin{tabular}{|c|c|c|}
\hline 24-h SBP (mmHg) & OR(95\%Cl) & $P$ value \\
\hline Unadjusted & $1.34(1.03-2.13)$ & 0.01 \\
\hline Adjusted & $1.13(0.78-2.13)$ & 0.11 \\
\hline \multicolumn{3}{|l|}{ 24-h DBP (mmHg) } \\
\hline Unadjusted & $1.42(1.21-2.11)$ & 0.002 \\
\hline Adjusted & $1.18(0.91-1.93)$ & 0.06 \\
\hline \multicolumn{3}{|c|}{ Daytime SBP,mmHg } \\
\hline Unadjusted & $1.33(1.04-2.35)$ & 0.01 \\
\hline Adjusted & $1.24(0.56-2.35)$ & 0.28 \\
\hline \multicolumn{3}{|c|}{ Daytime DBP (mmHg) } \\
\hline Unadjusted & $1.36(1.18-2.89)$ & 0.012 \\
\hline Adjusted & $1.31(0.89-2.27)$ & 0.15 \\
\hline \multicolumn{3}{|c|}{ Nighttime SBP,mmHg } \\
\hline Unadjusted & $2.01(1.53-3.58)$ & $<0.001$ \\
\hline Adjusted & $1.67(1.31-3.21)$ & $<0.001$ \\
\hline \multicolumn{3}{|c|}{ Nighttime DBP (mmHg) } \\
\hline Unadjusted & $1.28(1.32-3.01)$ & 0.02 \\
\hline Adjusted & $1.18(0.39-2.13)$ & 0.07 \\
\hline \multicolumn{3}{|c|}{ Night-to-day SBP ratio } \\
\hline Unadjusted & $2.47(1.98-3.87)$ & $<0.001$ \\
\hline Adjusted & $1.88(1.56-3.78)$ & $<0.001$ \\
\hline \multicolumn{3}{|c|}{ Night-to-day DBP ratio } \\
\hline
\end{tabular}




\begin{tabular}{|l|l|l|}
\hline Unadjusted & $1.76(1.07-2.59)$ & 0.06 \\
\hline Adjusted & $1.23(0.78-2.13)$ & 0.07 \\
\hline
\end{tabular}

Table 3: Association between Ambulatory Systolic Blood Pressure variables and hypertensive left ventricular hypertrophy.

\section{Abbreviations}

CI: Confidence Interval; OR: Odds Ratio.

\section{Discussion}

This is the cross-sectional first study to investigate the relations between ABPM and LVH in untreated hypertensive patients. In the present study, we found that nocturnal SBP the night to day systolic pressure ratio were independent risk factor of LVH in hypertensive patients.

The normal BP circadian rhythm typically presents nocturnal BP decrease by $10-20 \%$ compared with daytime BP on waking. However, previous study has shown that $13.2 \%$ of subjects with normotension have isolated nocturnal hypertension, and $25 \%$ to $35 \%$ of hypertensive patients have isolated nocturnal hypertension [17]. Moreover, Consistent studies have reported that isolated nocturnal hypertension was also associated with cardiac, vascular, renal, and cerebrovascular target organ damage [18], which may explain the consistent association between nocturnal increase in BP with substantial cardiac morbidity $[19,20]$. In the present study, we found the positive associations of nocturnal SBP with LVH. The pathophysiology underlying mechanism to explain this association of nocturnal hypertension and LVH remains uncertain. First, the nocturnal increase in BP is mainly due to the increase in sympathetic nervous activity and decrease in parsympathetic nervous activity during nighttime sleep. Consistent studies have indicated that catecholamine (norepinephrine and epinephrine) excretion was increased in untreated essential hypertensive patients with blunted nocturnal BP decline compared with dipper counterparts. Therefore, sympathetic activation in human hypertension may be responsible for circadian BP variations. The sympathetic nervous activity at night can further activate the rennin-angiotensin-aldosterone system (RAAS) in patients with hypertension, leading to a pronounced increase in secretion of Angiotensin II and aldosterone. Angiotensin II stimulates directly cardiac muscle cells and lead to myocardial hypertrophy. Aldosterone can induces myocardial fibrosis via production of increasing collagen, eventually leading to myocardial hypertrophy [21].

Night to day BP ratio is an important index of presenting circadian rhythm. Our study found that the night to day SBP ratio in hypertensive patients with $\mathrm{LVH}$ was higher than that in hypertensive patients without LVH. Further study found that night to day SBP ratio was also an independent risk factor of LVH in hypertension. Consistent study has been demonstrated that hypertensive patients with the increase of night to day blood pressure ratio could higher risks of cardiovascular events [22,23]. In addition, the relation between night blood pressure and left ventricular hypertrophy has been reported [24], but the strength of our present study must be considered compared with previous study. Firstly, all hypertensive patients were not taking antihypertensive drug, which excluded the effects of antihypertensive therapy on left ventricular hypertrophy. Secondly, all patients of our study were in-patients. Thus the clinical data including blood pressure and risk factor is more reliable. Thirdly, the echocardiographic examination was performed by the physician echocardiographers to determine LVH. The results of our study were more accurate and reliable. Moreover, previous study indicates that modification of drug-administration timing can alter the circadian rhythm of BP. The relationship between drug-modification in circadian rhythm of BP and cardiovascular events will be provided in further study.

\section{Conclusion}

The nocturnal SBP and the night to day SBP ratio are independent risk factors of $\mathrm{LVH}$ in hypertensive patients. The timing of drug administration in hypertensive patients needs to be further investigated by prospective studies.

\section{Limitations}

Several limitations must be considered. Firstly, the patients were initially diagnosed as hypertension. The duration of hypertension was unclear. The duration of hypertension were not assessed in the model of multiple regression. The power value may have been limited. Secondly, the research was a cross-sectional study and the causal relationship between the nocturnal systolic pressure and LVH was uncertain. Further prospective studies should clarify the influence of nocturnal blood pressure on LVH and related cardiovascular events. Thirdly, the sample of our present study was small, which can lead to a limited in statistical power. Thus further studies with a larger sample size are needed.

\section{Funding}

This work was partly supported by Chinese PLA General Hospital Clinical Sustaining Project (fund number 2012FC-TSYS-4003).

\section{References}

1. Rossignol P, Hosseini K, Tropeano AI, Fay R, Tsatsaris A, et al. (2013) Target organ damage assessment in French hypertensive patients without established cardiovascular or renal disease: results of the PREVENT-A study. J Hypertens 31: 177-185.

2. Nelissen HE, Hendriks ME, Wit FW, Bolarinwa OA, Osagbemi GK, et al. (2014) Target organ damage among hypertensive adults in rural Nigeria: a cross-sectional study. J Hypertens 32: 487-494.

3. Kavey RE (2013) Left ventricular hypertrophy in hypertensive children and adolescents: predictors and prevalence. Curr Hypertens Rep 15: 453-457.

4. Wang SX, Xue H, Zou YB, Sun K, Fu CY, et al. (2012) Prevalence and risk factors for left ventricular hypertrophy and left ventricular geometric abnormality in the patients with hypertension among Han Chinese. Chin Med J (Engl) 125: 21-26.

5. Peer M, Boaz M, Zipora M, Shargorodsky M (2013) Determinants of left ventricular hypertrophy in hypertensive patients: identification of highrisk patients by metabolic, vascular, and inflammatory risk factors. Int J Angiol 22: 223-228.

6. Vernooij JW, van der Graaf Y, Nathoe HM, Bemelmans RH, Visseren FL, et al. (2013) Hypertensive target organ damage and the risk for vascular events and all-cause mortality in patients with vascular disease. J Hypertens 31: 492-499.

7. Abad-Cardiel M, Alvarez-Álvarez B, Luque-Fernandez L, Fernández C, Fernández-Cruz A, et al. (2013) Hypertension caused by primary hyperaldosteronism: increased heart damage and cardiovascular risk. Rev Esp Cardiol (Engl Ed) 66: 47-52.

8. Angeli F, Reboldi G, Verdecchia P (2012) Microcirculation and leftventricular hypertrophy. J Hypertens 30: 477-481. 
Citation: Zhu H, Zhang W, Shi Y, Ma Y, Han Y, Liu J, Hao Xue (2014) Relationship between Nocturnal Blood Pressure and Left Ventricular Hypertrophy in Hypertensive Patients. J Hypertens 3: 151. doi:10.4172/2167-1095.1000151

Page 5 of 5

9. Mancia G, Zanchetti A, Agabiti-Rosei E, Benemio G, De Cesaris R, et al. (1997) Ambulatory blood pressure is superior to clinic blood pressure in predicting treatment-induced regression of left ventricular hypertrophy. SAMPLE Study Group. Study on Ambulatory Monitoring of Blood Pressure and Lisinopril Evaluation. Circulation 95: 1464-1470.

10. Wei FF, Li Y, Zhang L, Xu TY, Ding FH, et al. (2014) Association of target organ damage with 24-hour systolic and diastolic blood pressure levels and hypertension subtypes in untreated Chinese. Hypertension 63: 222-228.

11. Gómez-Marcos MA, Recio-Rodríguez JI, Patino-Alonso MC, AgudoConde C, Fernandez-Alonso C, et al. (2014) Electrocardiographic left ventricular hypertrophy criteria and ambulatory blood pressure monitoring parameters in adults. Am J Hypertens 27: 355-362.

12. Rodilla E, Costa JA, Martín J, González C, Pascual JM, et al. (2014) Impact of abdominal obesity and ambulatory blood pressure in the diagnosis of left ventricular hypertrophy in never treated hypertensives. Med Clin (Barc) 142: 235-242.

13. Guidelines Subcommittee (1999) 1999 World Health Orgnization international Society of Hypertension guidelines for the management of hypertension. J Hypertens 17: 151-183.

14. Levey AS, Coresh J, Greene T, Stevens LA, Zhang YL, et al. (2006) Using standardized serum creatinine values in the modification of diet in renal disease study equation for estimating glomerular filtration rate. Ann Intern Med 145: 247-254.

15. Fagard RH (2009) Dipping pattern of nocturnal blood pressure in patients with hypertension. Expert Rev Cardiovasc Ther 7: 599-605.

16. Devereux RB, Alonso DR, Lutas EM, Gottlieb GJ, Campo E, et al. (1986) Echocardiographic assessment of left ventricular hypertrophy: comparison to necropsy findings. Am J Cardiol 57: 450-458.
17. Li Y, Staessen JA, Lu L, Li LH, Wang GL, et al. (2007) Is isolated nocturnal hypertension a novel clinical entity? Findings from a Chinese population study. Hypertension 50: 333-339.

18. Routledge FS, McFetridge-Durdle JA, Dean CR; Canadian Hypertension Society (2007) Night-time blood pressure patterns and target organ damage: a review. Can J Cardiol 23: 132-138.

19. Ohkubo T, Hozawa A, Yamaguchi J, Kikuya M, Ohmori K, et al. (2002) Prognostic significance of the nocturnal decline in blood pressure in individuals with and without high 24-h blood pressure: the Ohasama study. J Hypertens 20: 2183-2189.

20. Cuspidi C, Giudici V, Negri F, Sala C (2010) Nocturnal nondipping and left ventricular hypertrophy in hypertension: an updated review. Expert Rev Cardiovasc Ther 8: 781-792.

21. Schlaich MP, Kaye DM, Lambert E, Sommerville M, Socratous F, et al. (2003) Relation between cardiac sympathetic activity and hypertensive left ventricular hypertrophy. Circulation 108: 560-565.

22. Yano Y, Kario K (2012) Nocturnal blood pressure and cardiovascular disease: a review of recent advances. Hypertens Res 35: 695-701.

23. Hansen TW, Li Y, Boggia J, Thijs L, Richart T, et al. (2011) Predictive role of the nighttime blood pressure. Hypertension 57: 3-10.

24. Iantorno M, Pola R, Schinzari F, Filice G, Mettimano M, et al. (2003) Association between altered circadian blood pressure profile and cardiac end-organ damage in patients with renovascular hypertension. Cardiology 100: 114-119. 